\title{
On the Galactic chemical evolution of sulfur
}

\author{
N. Ryde ${ }^{1,2}$ and D. L. Lambert ${ }^{2}$ \\ 1 Uppsala Astronomical Observatory, Box 515, 75120 Uppsala, Sweden \\ 2 Department of Astronomy, University of Texas, Austin, TX 78712-1083, USA
}

Received 21 May 2003 / Accepted 12 November 2003

\begin{abstract}
Sulfur abundances have been determined for ten stars to resolve a debate in the literature on the Galactic chemical evolution of sulfur in the halo phase of the Milky Way. Our analysis is based on observations of the S I lines at 9212.9, 9228.1, and $9237.5 \AA$ for stars for which the S abundance was obtained previously from much weaker S I lines at 8694.0 and $8694.6 \AA$. In contrast to the previous results showing $[\mathrm{S} / \mathrm{Fe}]$ to rise steadily with decreasing $[\mathrm{Fe} / \mathrm{H}]$, our results show that $[\mathrm{S} / \mathrm{Fe}]$ is approximately constant for metal-poor stars $([\mathrm{Fe} / \mathrm{H}] \lesssim-1)$ at $[\mathrm{S} / \mathrm{Fe}] \simeq+0.3$. Thus, sulfur behaves in a similar way to the other $\alpha$ elements, with an approximately constant $[\mathrm{S} / \mathrm{Fe}]$ for metallicities lower than $[\mathrm{Fe} / \mathrm{H}] \simeq-1$. We suggest that the reason for the earlier claims of a rise of $[\mathrm{S} / \mathrm{Fe}]$ is partly due to the use of the weak S I 8694.0 and $8694.6 \AA$ lines and partly uncertainties in the determination of the metallicity when using Fe I lines. The S I 9212.9, 9228.1, and $9237.5 \AA$ lines are preferred for an abundance analysis of sulfur for metal-poor stars.
\end{abstract}

Key words. stars: abundances - stars: atmospheres - stars: population II - Galaxy: abundances - Galaxy: evolution Galaxy: halo

\section{Introduction}

Galactic chemical evolution of the $\alpha$ elements $(\mathrm{O}, \mathrm{Ne}, \mathrm{Mg}, \mathrm{Si}$, $\mathrm{S}, \mathrm{Ar}$, and $\mathrm{Ca}$ ) might be expected to be similar from element to element. It is thought that their production in the early Galaxy was dominated by synthesis in massive stars and ejection into the interstellar medium by supernovae ( $\mathrm{SN}$ type II). Then, relative abundances of $\alpha$ elements and of their abundances with respect to iron reveal information about the yields from SN II and the dependence of those yields on the initial metallicity. After a passage of time, the Galactic gas was contaminated by ejecta of type Ia supernovae (exploding white dwarfs). These produce little of the $\alpha$ elements but large amounts of iron-group nuclei. Thus, the relative abundance of $\alpha$ elements to iron declined.

Accurate data on the abundances of $\alpha$ elements for stars in the halo and disk are essential if one is to extract information about the nucleosynthesis of these elements. First estimates of the $\mathrm{S}$ abundance in halo stars were provided by Takada-Hidai et al. (2002) and Israelian \& Rebolo (2001) who found that $[\mathrm{S} / \mathrm{Fe}]$ increased approximately linearly with decreasing $[\mathrm{Fe} / \mathrm{H}]:[\mathrm{S} / \mathrm{Fe}] \simeq-0.4 \times[\mathrm{Fe} / \mathrm{H}]$ from the Sun $([\mathrm{Fe} / \mathrm{H}] \equiv 0)$ to $[\mathrm{Fe} / \mathrm{H}] \simeq-3$, the metallicity limit of the samples. In contrast, Nissen et al. (2003a) provide strong evidence for a constant $[\mathrm{S} / \mathrm{Fe}] \simeq 0.35$ for stars with $[\mathrm{Fe} / \mathrm{H}] \lesssim-1$, and a quasi-linear rise to this value from $[\mathrm{Fe} / \mathrm{H}]=0$. This discrepancy among the observational results for $[\mathrm{Fe} / \mathrm{H}] \lessgtr-1$ deserves to be resolved before $\mathrm{S}$ abundances are added to the

Send offprint requests to: N. Ryde, e-mail: ryde@astro.uu. se observational inventory relevant to interpretations of Galactic chemical evolution. This paper describes our attempt to resolve the discrepancy.

A problem with obtaining the sulfur abundance of metalpoor stars is the paucity of suitable atomic lines. Candidate lines of S I lie in the near-infrared (NIR): multiplets of high-excitation lines occur near $8694 \AA$ and at 9212-9238 $\AA$. The drawback with the lines at $8694.0 \AA$ and $8694.6 \AA$ is their weakness in halo stars. Israelian \& Rebolo (2001) and Takada-Hidai et al. (2002) observed these lines. On the other hand, a disadvantage with the much stronger triplet at $9212.9,9228.1$, and $9237.5 \AA$ is the heavy interference by the telluric $\mathrm{H}_{2} \mathrm{O}$ lines. Nissen et al. (2003a) observed these $\lambda \lambda 9213-38$ lines and corrected for the $\mathrm{H}_{2} \mathrm{O}$ line absorption.

In this project, we chose to observe the $\lambda \lambda 9213-38$ lines in a number of the stars observed at $\lambda \lambda 8694-95$ by Takada-Hidai et al. (2002, in the following shortened as TH) and Israelian \& Rebolo (2001, abbreviate as I\&R). Our stars sample the metallicity range down to $[\mathrm{Fe} / \mathrm{H}] \simeq-3$, and consist of three from the sample of I\&R, three from TH, and four from François (1988), whose equivalent widths were reanalyzed by TH and I\&R. Our primary goal was to check the high $[\mathrm{S} / \mathrm{Fe}]$ reported previously from the $\lambda \lambda 8694-95$ lines. This check is executed using high quality spectra of the $\lambda \lambda 9213-38$ lines analyzed using model atmospheres computed for the same effective temperature and surface gravity as used in the pioneering studies from which our star-list is made. As a secondary goal, we sought to gain an 
indication of how $[\mathrm{S} / \mathrm{Fe}]$ behaves among halo stars of different metallicities.

\section{Observations}

The investigated stars, all lying in the northern sky, are presented in Table 1. I\&R determined the sulfur abundance for 8 new stars, including two upper limits, with the $4.2 \mathrm{~m}$ William Herschel Telescope on La Palma. We have analyzed three of these, namely HD 2665, HD 19445, and HD 201889. TH made new determinations of the sulfur abundance in 6 stars (with one upper limit) using the HIRES spectrograph on the Keck telescope on Hawaii. Of these we have observed three (HD 84937, HD 88609, and HD 165195). Furthermore, we have observed HD 111721, HD 94028, HD 132475, and HD 201891, which were originally observed and analyzed by François (1988), and then reanalyzed both by TH and I\&R.

The stars, which span the metallicity range of $-2.75 \lesssim$ $[\mathrm{Fe} / \mathrm{H}]^{1} \lesssim-0.7$, were observed in observing runs from November 29 and 30, 2001 and April 27, 2002 at the W. J. McDonald Observatory using the 2.7-m Harlan J. Smith telescope with the high-resolution 2dcoudé cross-dispersed echelle spectrograph (Tull et al. 1995). The resolving power was approximately $R=60000$, a value determined from the FWHM of thorium lines in the wavelength calibration frames. Full spectral coverage from 3400 to $10900 \AA$ can be obtained in two exposures. However, we observed every star in only one setting of the echelle, thus retrieving the entire wavelength range but with gaps. Our bandpass includes the sulfur $\lambda \lambda 9213-38$ lines but not the $\lambda \lambda 8694-95$ lines. We used the TK3 detector, a $2048 \times 2048$ pixel $^{2}$ CCD with $24 \mu$ m pixels. The observing times, ranging from a half-an-hour to 3 hours for the F, G, and $\mathrm{K}$ stars, are given in Table 1 . To avoid a large number of cosmic hits, individual exposures were limited to a maximum of $30 \mathrm{~min}$.

The observed CCD data was processed with the reduction package IRAF to retrieve one-dimensional, continuum normalized, and wavelength calibrated, pure stellar spectra. In the wavelength calibration a root-mean-square of the fit of less than $4 \mathrm{~m} \AA$ is achieved. Multiple exposures of the same star were added in the form of 1-dimensional spectra by weighting the different spectra by their mean count levels. When several frames are available a cosmic-ray-hit rejection algorithm was applied.

The local continua of the F, G, and K star spectra were fitted and normalized by a low-order Legendre function in order not to remove the wings of the stellar Paschen line. As can be seen in Fig. 1, one of the sulfur lines lies in the wing of this hydrogen line. The hydrogen lines in the dwarf spectra have wings stretching over a considerable wavelength range and we therefore have to proceed with caution when fitting the continuum.

The $9200 \AA$ region of interest here is marred by numerous telluric absorption lines (Fig. 1). To eliminate the telluric contribution to the spectra, we observed fast-rotating-B-stars

\footnotetext{
1 The bracket notation is defined as follows: $[A / B] \equiv \log (A / B)_{\star}-$ $\log (A / B)_{\odot}$.
}

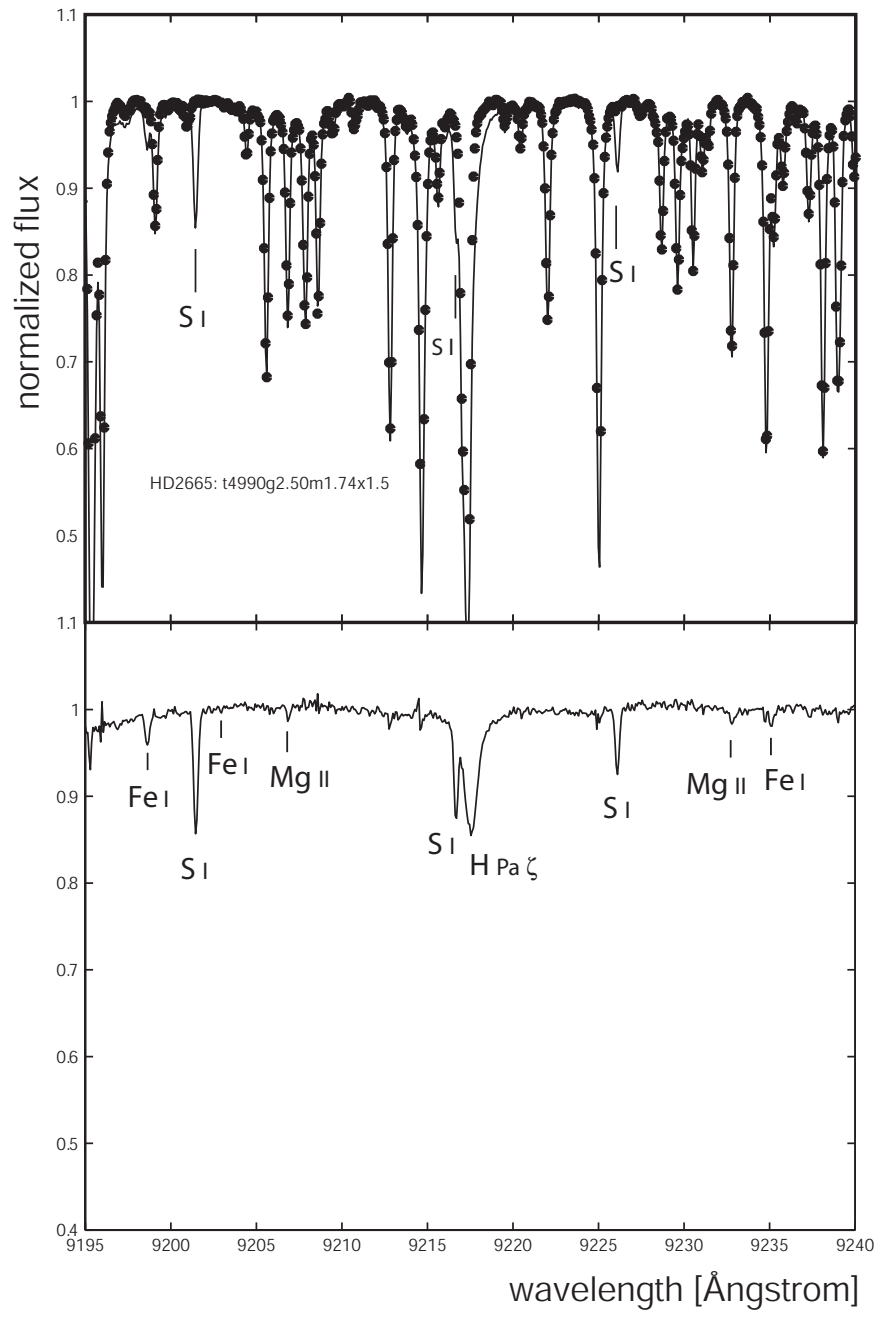

Fig. 1. In the upper panel, the full line displays the continuumnormalized spectrum of our giant star HD 2665 (including telluric lines, mostly water vapor lines) and, as dots, the continuumnormalized spectrum of our rapidly rotating B-star, which shows only telluric absorption lines. In the lower panel, the HD 2665 spectrum is divided by this telluric spectrum, resulting in a pure stellar spectrum of HD 2665. The sulfur lines and the Paschen $\zeta$ hydrogen line emerge clearly after the division. Magnesium and iron lines are also marked. The abscissa and ordinate scales are the same in both panels. Observe that the local continuum of the HD 2665 spectrum is fitted with a low order function, to avoid eliminating the wings of the hydrogen line. This will be even more important for the dwarfs.

with $v \sin i=150-350 \mathrm{~km} \mathrm{~s}^{-1}$. The intrinsic, B-star spectra are featureless except for a more or less pronounced, rotationally broadened Paschen $\zeta \mathrm{H}$ I line at $9229.02 \AA$, which means that they can be used as a template for the telluric absorption lines. For these B-star spectra, we used a spline function of high order to fit and normalize the spectra, but also to eliminate the broad Paschen $\zeta$ HI feature and occurring fringes. The hot stars were observed close in time and at a similar airmass as the programme stars, and were required to have a signal-to-noise ratio of several hundred. To achieve this in the $9200 \AA$ region, exposure times from tens of seconds up to an hour were required, depending on the brightness of the individual star and the specific spectral type. The reduced B-star spectra achieved in 
Table 1. Investigated stars.

\begin{tabular}{llcccccccc}
\hline \hline Star & $\begin{array}{l}\text { Spectral } \\
\text { type }^{a}\end{array}$ & $\begin{array}{c}V \\
{[\mathrm{mag}]}\end{array}$ & $\begin{array}{c}T_{\text {eff }} \\
{[\mathrm{K}]}\end{array}$ & $\begin{array}{c}\log (g) \\
(\mathrm{cgs})\end{array}$ & {$[\mathrm{Fe} / \mathrm{H}]$} & $\begin{array}{c}\xi_{\text {micro }} \\
{\left[\mathrm{km} \mathrm{s}^{-1}\right]}\end{array}$ & $\begin{array}{c}\xi_{\text {macro }} \\
{\left[\mathrm{km} \mathrm{s}^{-1}\right]}\end{array}$ & $\begin{array}{c}\text { Date of } \\
\text { observations }\end{array}$ & $\begin{array}{c}\text { Exposure time } \\
{[\mathrm{s}]}\end{array}$ \\
\hline Giants and subgiants & & & & & & & & & \\
HD 2665 & G5III & 7.8 & $4990 \pm 70$ & $2.50 \pm 0.2$ & $-1.74 \pm 0.15$ & $1.5 \pm 0.5$ & $5.5 \pm 0.5$ & Nov. 29, 2001 & 7200 \\
HD 88609 & G5IIIw & 8.6 & $4570 \pm 100$ & $0.75 \pm 0.15$ & $-2.85 \pm 0.15$ & $1.9 \pm 0.5$ & $7.0 \pm 0.5$ & Apr. 27, 2002 & 3600 \\
HD 111721 & G6V & 8.0 & $5010 \pm 100$ & $2.31 \pm 0.15$ & $-1.27 \pm 0.15$ & $1.2 \pm 0.5$ & $4.5 \pm 0.5$ & Apr. 27, 2002 & 3600 \\
HD 165195 & K3p & 7.3 & $4190 \pm 100$ & $1.00 \pm 0.15$ & $-1.75 \pm 0.15$ & $1.3 \pm 0.5$ & $7.5 \pm 0.5$ & Apr. 27, 2002 & 1800 \\
\hline Dwarfs & & & & & & & & & \\
HD 19445 & sdG5 & 8.0 & $5810 \pm 150$ & $4.46 \pm 0.2$ & $-1.90 \pm 0.15$ & $1.5 \pm 0.5$ & $4.5 \pm 0.5$ & Nov. 29, 2001 & 10800 \\
HD 84937 & sdF5 & 8.3 & $6300 \pm 100$ & $3.97 \pm 0.15$ & $-2.06 \pm 0.15$ & $1.1 \pm 0.5$ & $5.0 \pm 0.5$ & Nov. 30, 2001 & 9000 \\
HD 94028 & F4V & 8.2 & $5980 \pm 100$ & $4.30 \pm 0.15$ & $-1.35 \pm 0.15$ & $1.5 \pm 0.5$ & $4.5 \pm 0.5$ & Apr. 27, 2002 & 3600 \\
HD 132475 & F5/F6V & 8.6 & $5810 \pm 100$ & $3.91 \pm 0.15$ & $-1.38 \pm 0.15$ & $1.9 \pm 0.5$ & $4.5 \pm 0.5$ & Apr. 27, 2002 & 3600 \\
HD 201891 & F8V - VI & 7.4 & $5880 \pm 100$ & $4.25 \pm 0.15$ & $-1.03 \pm 0.15$ & $1.5 \pm 0.5$ & $4.0 \pm 0.5$ & Apr. 27, 2002 & 1800 \\
HD 201889 & G1V & 8.0 & $5615 \pm 100$ & $4.24 \pm 0.2$ & $-0.71 \pm 0.15$ & $1.5 \pm 0.5$ & $4.0 \pm 0.5$ & Apr. 27, 2002 & 1800 \\
\hline
\end{tabular}

a From Simbad (http://simbad.u-strasbg.fr).

this manner are assumed to contain purely telluric absorption lines. The programme spectra were subsequently divided by a scaled version of the "telluric" spectra. The divided, reduced spectra are shown in Figs. 2 and 3. As can be seen in these figures, the telluric lines are astonishingly well eliminated while the stellar sulfur lines show up clearly. While our procedure of eliminating the telluric lines adds to the noise, the resultant signal-to-noise ratio of the ratioed stellar-spectra is satisfactory for our purpose.

The equivalent widths of the sulfur lines at $9212.9 \AA$ and $9237.5 \AA$, which are considered to be unblended, were measured by Gaussian fits or by total integration of the line. The equivalent width of the $9228.1 \AA$ line is not measured (except for HD 111721) since it lies in the stellar Paschen $\zeta$ HI line. The measured equivalent widths of the two (three) sulfur lines, the equivalent widths of the SI $8694.6 \AA$ line supplied in the literature (I\&R; TH), and our measured equivalent widths of six Fe II lines (lying at 4416.8, 5264.8, 5284.1, 6149.3, 6432.7, and $6456.4 \AA$ ) are presented in Table 2 . We note that the measured equivalent widths of the sulfur lines at 9212.9, (9228.1), and $9237.5 \AA$ range from approximately 10 to $100 \mathrm{~m} \AA$, which is suitable for a reliable abundance analysis. Observe also, that the equivalent widths are an order of magnitude larger than those determined for the line at 8694.6 $\mathrm{A}$. The 8694.0 $\AA$ line is a factor of five weaker still and unmeasurable in all the metalpoor stars we have been investigating, except HD 201889.

We note that the TK3 detector used consists of a thick chip, which is not severely affected by fringing. The existing fringing cancels well using a flat field - the top panel is Fig. 1 compares two such flat-fielded spectra. Fringing is eliminated to a high degree by using a hot star observed at the same time and the lower panel of Fig. 1 shows just such a spectrum.

In Figs. 2 and 3, there exist low points which originate from the cancellation of the very strongest telluric lines. None of these affect the SI lines. For example, in the spectrum of HD 19445 in Fig. 3 only the $9212.9 \AA$ line lies in a wing of a modestly strong telluric line. The other ones are free. The abundance derived from the free $9237.5 \AA$ line is $\log A_{\mathrm{S}}=5.51$ (the logarithmic number-abundance of sulfur relative to hydrogen, $\log A_{\mathrm{S}}=\log N_{\mathrm{S}} / N_{\mathrm{H}}$, normalized on a scale where $\log A_{\mathrm{H}}=12.00$ ), whereas the ratioed $9212.9 \AA$ Alne gives 5.49. The synthetic spectrum which fits these two lines, as well as the $9228.1 \AA$ line is calculated with an abundance of 5.50. The fact that the three sulfur lines are synthesised well with one sulfur abundance shows that the elimination of the telluric lines produces a reliable result, clearly within the other uncertainties. We estimate the error in the equivalent widths to be less than 5\%, implying an uncertainty in the derived sulfur abundance of the order of $0.05 \mathrm{dex}$, as estimated from the individual measurements.

In some regions, for example around $9207 \AA$ in the spectrum of HD 111721 in Fig. 2 and around $9245 \AA$ in the spectrum of HD 132475 in Fig. 3, the elimination of the telluric lines is hampered due to closely lying telluric lines with wings blending with each other. The equivalent widths of the sulfur lines are, however, measured by drawing a local continuum which should reduce systematic errors due to this effect.

\section{Analysis}

We analyze our data by modelling the stellar atmosphere and requiring that the measured equivalent widths are reproduced for the two unblended S I $\lambda 9213$ and $\lambda 9238$ lines. The mean sulfur abundance yielded in this way is subsequently used in calculating a synthetic spectrum for a given atmosphere of the entire region (9200-9500 $\AA$ ). The synthetic spectrum is thereafter convolved with a macroturbulence function in order to fit the shapes and widths of the lines, see Figs. 2 and 3. In this section we will discuss the model atmospheres, the stellar parameters including uncertainties, the line data, and the spectrum synthesis. 

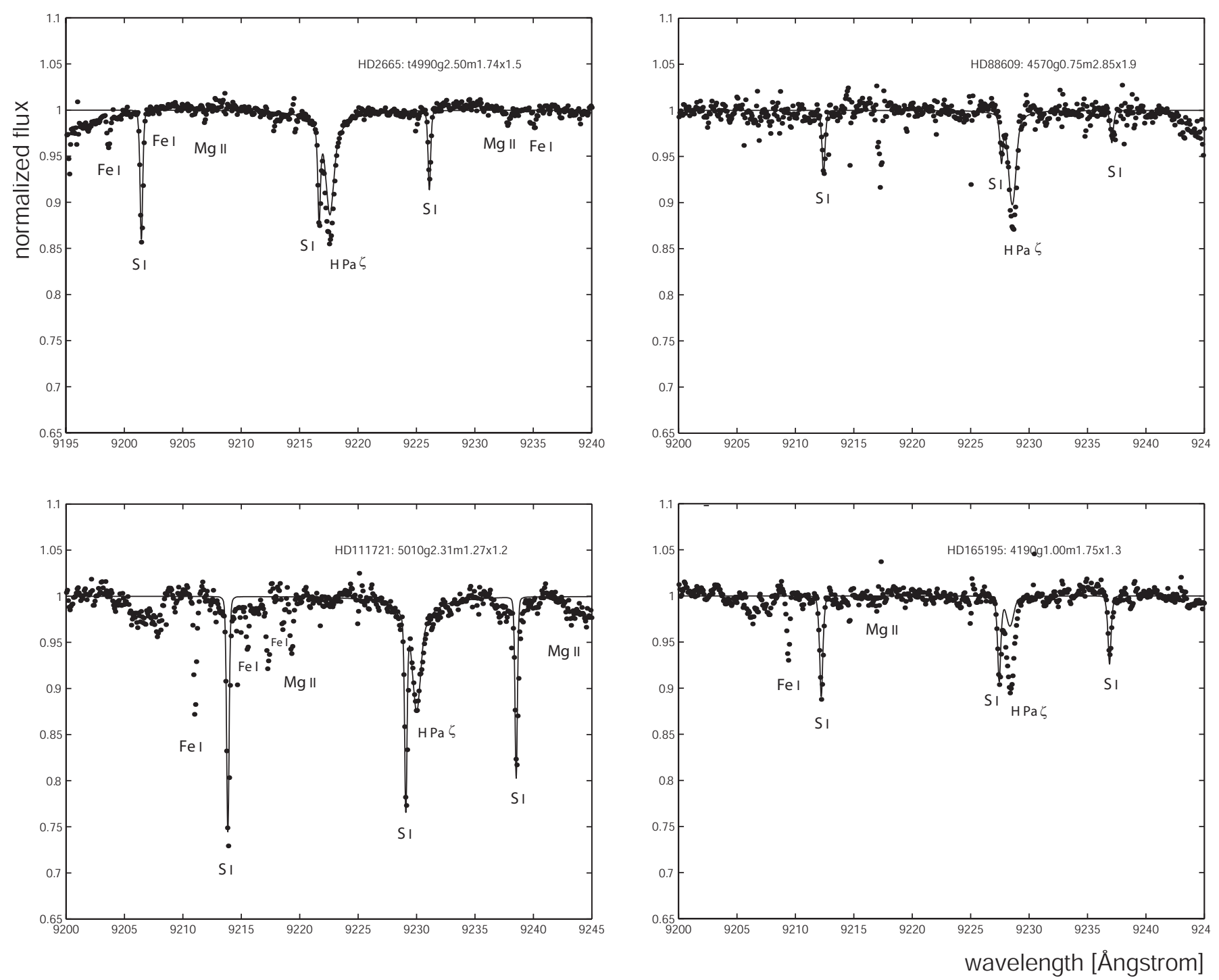

Fig. 2. Continuum-normalized spectra of our programme giants: HD 2665, HD 88609, HD 111721, and HD 165195. Telluric absorption lines are ratioed out, but some residual signatures are visible. In the spectra of HD 2665 and HD 111721 several Fe I lines and a Mg II line are discernable and in the spectrum of HD 165195 the most conspicuous Fe I line is detected. In the lower right panel in Fig. 3 identifications of all observable metal lines in this wavelength region can be found. Our best model spectra are also plotted with full lines. See text for a comment on the bad fit of the hydrogen Paschen line in the spectrum of HD 165195. After the names the star-model's temperature, log $g$, metallicity, and microturbulence are indicated.

\subsection{Model atmospheres}

I\&R and TH used ATLAS9 model atmospheres whereas we use model atmospheres provided by the MARCS code. The MARCS code was first developed by Gustafsson et al. (1975) and has been successively updated ever since. These hydrostatic, planeparallel model photospheres are computed on the assumptions of Local Thermodynamic Equilibrium (LTE), homogeneity and the conservation of the total flux (radiative plus convective; the convective flux being computed using the mixing length formulation). Data on absorption by atomic species are collected from the VALD database (Piskunov et al. 1995) and Kurucz (1995, private communication). Absorption by molecules is included but quite unimportant for our stars.

We have computed model atmospheres in plane-parallel geometry which is an excellent approximation for the dwarfs. To test the validity of the approximation for the giant stars we calculated a model atmosphere in spherical geometry for the giant HD 88609 and compared it with a plane-parallel model. The equivalent widths of the sulfur lines are changed by only a few percent. We have, therefore, calculated model atmospheres for all our stars in plane-parallel geometry.

In our model atmospheres the enhancement of $\mathrm{C}, \mathrm{O}, \mathrm{Ne}$, $\mathrm{Mg}, \mathrm{Si}, \mathrm{S}, \mathrm{Ar}, \mathrm{Ca}$, and $\mathrm{Ti}$ (that is the $\alpha$ elements) was assumed to be $[\alpha / \mathrm{Fe}]=0.4$ except for HD 201889 for which it was assumed to be $[\alpha / \mathrm{Fe}]=0.2$, due to its higher metallicity. The derived $\mathrm{S}$ abundance is quite insensitive to the $\alpha$ enhancement.

Using their equivalent widths, line parameters and set of fundamental stellar parameters, we are able to reproduce the derived abundances in I\&R and TH. Our use of MARCS model atmospheres lowers the abundances by a few hundredths of a 

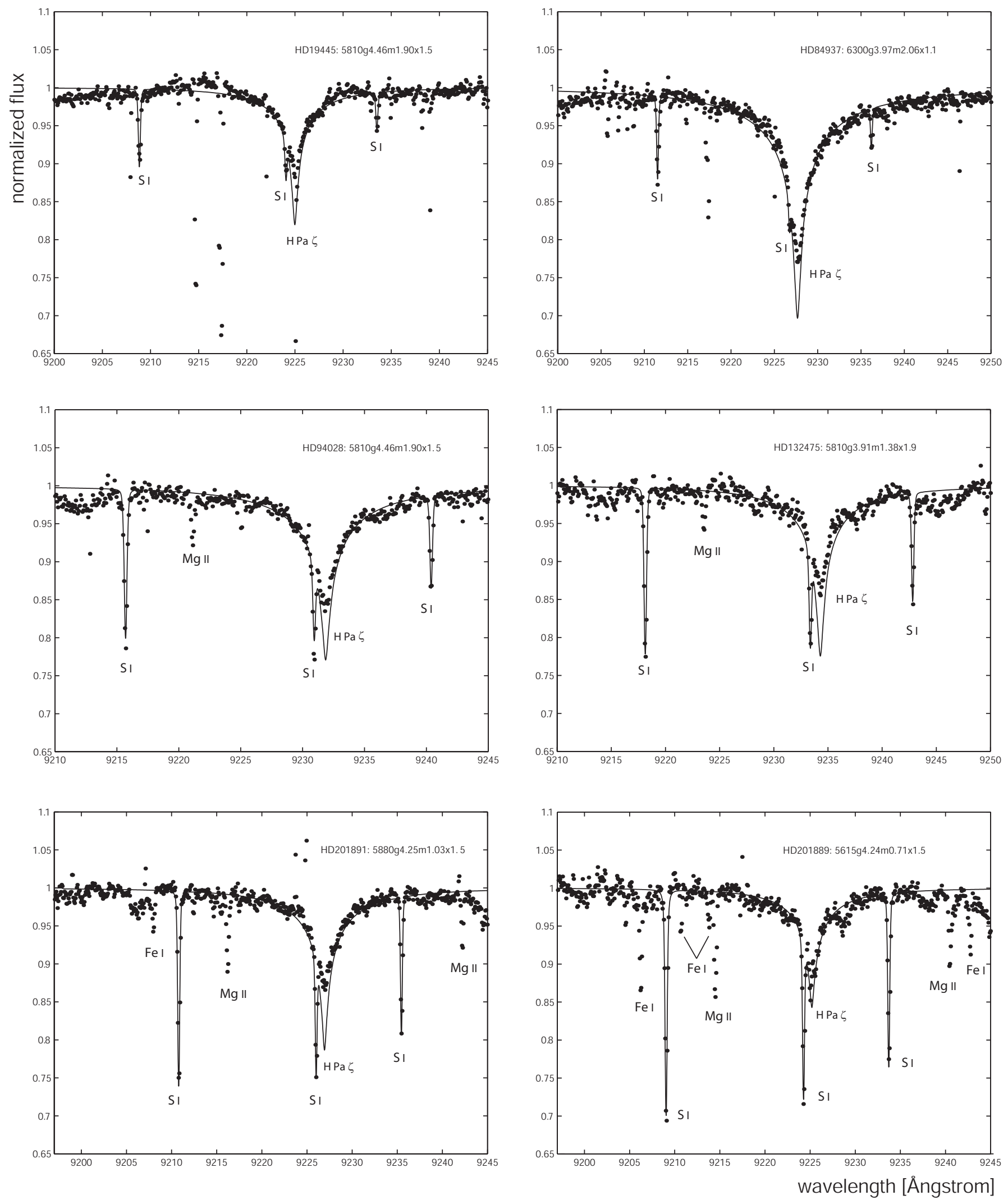

Fig. 3. Continuum normalized spectra of our programme dwarfs: HD 19445, HD 84937, HD 94028, HD 132475, HD 201891 , and HD 201889. The telluric absorption lines are removed as well as possible, but some residuals remain, most prominently in the spectrum of HD 19445 and HD 84937. Metal lines have been identified in the spectra of the four lowest panels. In the spectrum of the most metal-rich star, HD 201889, all the identified Fe I, Mg II, and S I lines are marked. The full lines represent our modelled spectra. After the names the star-model's temperature, $\log g$, metallicity, and microturbulence are indicated. 
Table 2. Measured equivalent widths given in $\mathrm{m} \AA$.

\begin{tabular}{lcccccccccc}
\hline \hline Star & $\begin{array}{c}W_{\text {S I }} \\
\text { 9212.9 }\end{array}$ & $\begin{array}{c}W_{\text {SI }} \\
9228.1 \AA\end{array}$ & $\begin{array}{c}W_{\text {SI }} \\
9237.5 \AA\end{array}$ & $\begin{array}{c}W_{\text {SI }} \\
8694.6 \AA\end{array}$ & $\begin{array}{c}W_{\text {FeII }} \\
4416.8 \AA\end{array}$ & $\begin{array}{c}W_{\text {FeII }} \\
5264.8 \AA\end{array}$ & $\begin{array}{c}W_{\text {FeII }} \\
5284.1 \AA\end{array}$ & $\begin{array}{c}W_{\text {FeII }} \\
6149.3 \AA\end{array}$ & $\begin{array}{c}W_{\text {FeII }} \\
6432.7 \AA\end{array}$ & $\begin{array}{c}W_{\text {FeII }} \\
6456.4 \AA\end{array}$ \\
\hline \multicolumn{2}{l}{ Giants and subgiants } & & & & & & & & & \\
HD 2665 & 45 & - & 22 & $3.0^{a}$ & - & 15 & 26 & 7.3 & 13 & 25 \\
HD 88609 & 24 & - & 13 & $<2.2^{b}$ & 32 & 7.0 & 13 & 2.7 & 5.7 & 11 \\
HD 111721 & 78 & 61 & 54 & $4.5^{c}$ & 73 & 28 & 40 & 16 & 24 & 41 \\
HD 165195 & 40 & - & 28 & $2.0^{b}$ & 65 & 20 & 36 & 9.0 & 18 & 29 \\
\hline Dwarfs & & & & & & & & & & \\
HD 19445 & 30 & - & 15 & $2.7^{a}$ & 13 & 2.0 & 4.5 & 1.3 & 1.5 & 5.7 \\
HD 84937 & 38 & - & 14 & $3.4^{b}$ & 14 & 1.8 & 3.7 & 1.2 & 2.1 & 5.8 \\
HD 94028 & 64 & - & 37 & $4.5^{c}$ & 33 & 7.2 & 13 & 5.4 & 8.5 & 18 \\
HD 132475 & 67 & - & 40 & $4.2^{c}$ & 37 & 9.1 & 17 & 6.7 & 9.8 & 26 \\
HD 201891 & 77 & - & 52 & $7.1^{c}$ & 46 & 14 & 23 & 11 & 12 & 31 \\
HD 201889 & 104 & - & 70 & $14^{a}$ & 54 & 27 & 35 & 17 & 19 & 42 \\
\hline
\end{tabular}

${ }^{a}$ From Israelian \& Rebolo (2001).

$b$ From Takada-Hidai et al. (2002).

${ }^{c}$ From François (1988).

dex relative to those obtained from ATLAS9 models used previously. Clearly, the choice of ATLAS9 vs. MARCS is not an important factor affecting the derived abundances.

\subsection{Stellar parameters}

We have chosen the same stellar, fundamental parameters for the model atmospheres (actually $T_{\text {eff }}, \log (g)$, and $\xi_{\text {micro }}$ ) as those determined in I\&R and TH. However, the metallicities of the stars $([\mathrm{Fe} / \mathrm{H}])$ we redetermined from six singly ionized iron lines. The fundamental stellar parameters including our determined metallicities and are given in Table 1.

As was noted above, the stars HD 111721, HD 94028, HD 132475, and HD 201891, which were originally observed and analyzed by François (1988), have been reanalyzed both by TH and I\&R. For the determination of the effective temperatures and surface gravity, TH took into account interstellar reddening which resulted in noticeable adjustments of the parameters. We will use parameters determined by $\mathrm{TH}$ for the François (1988) stars.

TH determined the effective temperatures of HD 84937 , HD 88609, HD 165195, and HD 111721 from the Alonso et al. (1999a) calibration, based on the infrared flux method (IRFM), also taking the interstellar reddening into account. For the reanalysis of the dwarfs HD 94028, HD 132475, and HD 201891, TH used the empirical temperature scale for dwarfs as formulated by Alonso et al. (1996). Our new metallicities do not alter this temperature determination significantly.

Furthermore, TH calculated the surface gravity using Hipparcos parallaxes. The microturbulence was determined for HD 84937, HD 88609, and HD 165195 from Fe I lines. Microturbulence for HD 111721 is taken from Ryan \& Lambert (1995), and for the dwarfs HD 94028, HD 132475, and
HD 201891 it was calculated from the empirical formula provided by Edvardsson et al. (1993).

For the giant star HD 2665, the effective temperature was determined by I\&R based on the IRFM in Alonso et al. (1999b) and the surface gravity $(\log g)$ from a non-LTE study of iron (for references see I\&R). For HD 19445 and HD 201889 the temperature is based on the IRFM (Israelian et al. 1998), and $\log g$ from an non-LTE analysis of iron by Thévenin \& Idiart (1999). The microturbulence we have assumed to be $\chi$ micro $=$ $(1.5 \pm 0.5) \mathrm{km} \mathrm{s}^{-1}$ for these three stars.

\subsection{Line data}

The high-excitation $(\chi=6.5 \mathrm{eV}) \mathrm{S}$ I lines used in this paper are due to the multiplet $4 s^{5} S^{0}-4 p^{5} \mathrm{P}$, while the lines at 8694.0 and $8694.6 \AA$ are from the multiplet $4 \mathrm{p}^{5} \mathrm{P}-4 \mathrm{~d}^{5} \mathrm{D}^{0}$. The line parameters (wavelength, excitation energy, line strength) of our S I lines and six Fe II lines are provided in Table 3.

In the following, we have used a solar sulfur abundance of $\log \varepsilon_{\odot}(\mathrm{S})=7.20$ (Chen et al. 2002) as a reference point.

\subsection{Spectral synthesis}

The sulfur abundance is originally found by reproducing the equivalent widths of the unblended sulfur $\lambda 9213$ and $\lambda 9238$ lines. This is done with the radiative transfer routines of the MARCS codes and yields a mean sulfur abundance with a standard deviation of 0.01 to 0.1 dex. (This gives an assessment of the random measuring uncertainties.) Synthetic spectra around $9200-9250 \AA$ are produced by computations of the radiative transfer through the model atmospheres, using the mean sulfur abundance and using our line data for all three sulfur lines and the Paschen $\zeta$ HI line. We calculate the radiative transfer for points in the spectrum separated by 
Table 3. Line parameters.

\begin{tabular}{rrr}
\hline \hline $\begin{array}{r}\text { Wavelength } \\
(\AA)\end{array}$ & $\begin{array}{r}E_{\text {exc }} \\
(\mathrm{eV})\end{array}$ & $\begin{array}{r}\log g f \\
(\mathrm{cgs})\end{array}$ \\
\hline S I LINES & & \\
9212.863 & 6.525 & $0.43^{a}$ \\
9228.093 & 6.525 & $0.25^{a}$ \\
9237.538 & 6.525 & $0.04^{a}$ \\
\hline Fe II LINES & & \\
4416.830 & 2.778 & $-2.80^{b}$ \\
5264.812 & 3.230 & $-3.19^{b}$ \\
5284.109 & 2.891 & $-3.29^{b}$ \\
6149.258 & 3.889 & $-2.82^{b}$ \\
6432.680 & 2.891 & $-3.67^{c}$ \\
6456.383 & 3.903 & $-2.25^{c}$ \\
\hline
\end{tabular}

a Line data taken from the NIST database.

b From Thorén et al. in preparation.

c From Thorén \& Feltzing (2000).

$\Delta \lambda \sim 0.6 \mathrm{~km} \mathrm{~s}^{-1}$ (corresponding to a resolution of $\lambda / \Delta \lambda \sim$ 460000 ). We subsequently convolve the spectra with a macrobroadening function, assuming a Doppler-shift distribution for both radial and tangential velocity components as specified by the "radial-tangential" model for the macroturbulence, for details see Gray (1992). The FWHM velocities for the macroturbulence broadening are given in Table 1 and were derived by requiring that the shape and widths of the sulfur lines should match the observations.

Note, that the sulfur $\lambda 9228$ line, which lies in the Paschen $\zeta$ HI line, will also be calculated (including the hydrogen opacity) by the synthetic spectrum program. For a few of the dwarfs the wings of the Paschen $\zeta$ HI line also interfere, to various extents, with the two sulfur lines which were assumed to be unblended. This means that the measured equivalent widths could be wrong in these cases, underestimating the sulfur abundance. Therefore, the sulfur abundances were adjusted in order that the synthetic spectrum, including the hydrogen-line wings, should fit the observations. The size of these changes were, however, not more than approximately $0.05 \mathrm{dex}$ in the sulfur abundance.

In Figs. 2 and 3 we have plotted the final synthetic spectra together with our observations. The modelled spectra are shifted according to the observed radial velocities of the stars. From the figures, we see that all sulfur lines are synthesized convincingly. Thus, the information from all three sulfur lines has been used in determining the sulfur abundance. The synthetic spectra only take the sulfur lines and the hydrogen line into account. The other metal lines in the region were not included in the calculation.

The calculations of the wings of the hydrogen line yield a satisfactory match to the observations, which can be seen, in particular, in the dwarf spectra (see Fig. 3). Recall that the continuum normalization was made with a low order function only, in order to fit the continuum over a limited range, so spurious features (such as possible residual fringing) are not taken out. The hydrogen line cores are expected to be subject to
Table 4. Effects on logarithmic abundances derived when changing the fundamental parameters of the model atmospheres. Two stars of different parameters are presented. The parameters $\left(T_{\text {eff }} / \log g /[\mathrm{Fe} / \mathrm{H}] / \xi_{\text {micro }}\right)$ are, respectively: $(4190 / 1.0 /-1.75 / 1.3)$ and $(6300 / 3.97 /-2.06 / 1.1)$.

\begin{tabular}{|c|c|c|c|}
\hline Star & Uncertainty & $\Delta[\mathrm{S} / \mathrm{H}]$ & $\Delta[\mathrm{S} / \mathrm{Fe}]$ \\
\hline \multirow[t]{4}{*}{ HD 165195} & $\delta T_{\text {eff }}=+100 \mathrm{~K}$ & -0.17 & -0.08 \\
\hline & $\delta \log g=+0.2(\mathrm{cgs})$ & +0.10 & +0.01 \\
\hline & $\delta[\mathrm{Fe} / \mathrm{H}]=+0.15$ & +0.06 & +0.01 \\
\hline & $\delta \xi_{\text {micro }}=+0.5$ & -0.12 & -0.03 \\
\hline \multirow[t]{4}{*}{ HD 84937} & $\delta T_{\text {eff }}=+100 \mathrm{~K}$ & -0.03 & -0.04 \\
\hline & $\delta \log g=+0.2(\mathrm{cgs})$ & +0.06 & -0.01 \\
\hline & $\delta[\mathrm{Fe} / \mathrm{H}]=+0.15$ & \pm 0.00 & \pm 0.00 \\
\hline & $\delta \xi_{\text {micro }}=+0.5$ & -0.06 & -0.05 \\
\hline
\end{tabular}

departures from LTE, which could lead to poor fits. The unsatisfactory fit of the hydrogen-line core in the giant HD 165195 , for example, could be improved but would require higher temperatures, of the order of $400 \mathrm{~K}$.

\subsection{Uncertainties in the stellar parameters}

The uncertainties in the fundamental parameters are adopted from their sources: for HD 2665, HD 19445, and HD 201889 from I\&R; for HD 84937, HD 88609, and HD 165195 from TH; and for HD 111721, HD 94028, HD 132475, and HD 201891 from the reanalysis of the François (1988) data by TH, see Table 1. For HD 2665, HD 19445 and HD 201889, we assess the uncertainties in the microturbulence to be of the same order as estimated in TH. Furthermore, we judge the uncertainties in the macroturbulence to be of the same order, that is $\pm 0.5 \mathrm{~km} \mathrm{~s}^{-1}$. The uncertainties in our new determination of the metallicity is assessed to be less than \pm 0.15 dex.

The propagation of these uncertainties into the determination of the sulfur abundance is calculated by changing the fundamental parameters of the model atmosphere with the estimated uncertainties and then running the synthetic spectrum program to obtain the change in sulfur abundance for a given equivalent width. Table 4 shows the consequence of the uncertainties in the effective temperature, surface gravity, metallicity, and microturbulence on the $[\mathrm{S} / \mathrm{H}]$ and $[\mathrm{S} / \mathrm{Fe}]$ ratios for one giant and one dwarf. For the coolest stars (e.g. HD 165195), the dominant source of uncertainty is the $100 \mathrm{~K}$ estimate for the temperature error. The uncertainties in the sulfur abundance, due to temperature uncertainties, are of the order of 0.04 dex or less for the dwarfs, and for the giants, typically $0.1 \mathrm{dex}$.

The total uncertainty in the $[\mathrm{S} / \mathrm{Fe}]$ due to uncertainties in the fundamental parameters is, in general, of the order of 0.05 , but could be as high as 0.08 in special cases. This is the same conclusion as Nissen et al. (2003a) arrive at in their investigation of the sulfur abundance in solar-type stars. 
Table 5. Abundances of Fe II and S I, the latter based on the $\lambda \lambda 9213-38$ lines.

\begin{tabular}{|c|c|c|c|c|c|}
\hline Star & $\log A_{\mathrm{Fe} \mathrm{II}}$ & {$[\mathrm{Fe} \mathrm{II} / \mathrm{H}]$} & $\log A_{\mathrm{S}}$ & {$[\mathrm{S} / \mathrm{H}]$} & {$[\mathrm{S} / \mathrm{Fe}]$} \\
\hline \multicolumn{6}{|c|}{ Giants and subgiants } \\
\hline HD 2665 & 5.76 & -1.74 & 5.60 & -1.60 & 0.14 \\
\hline HD 88609 & 4.65 & -2.85 & 4.74 & -2.46 & 0.39 \\
\hline HD 111721 & 6.23 & -1.27 & 6.25 & -0.95 & 0.32 \\
\hline HD 165195 & 5.75 & -1.75 & 5.97 & -1.23 & 0.52 \\
\hline \multicolumn{6}{|l|}{ Dwarfs } \\
\hline HD 19445 & 5.60 & -1.90 & 5.50 & -1.70 & 0.20 \\
\hline HD 84937 & 5.44 & -2.06 & 5.27 & -1.93 & 0.13 \\
\hline HD 94028 & 6.15 & -1.35 & 5.93 & -1.27 & 0.08 \\
\hline HD 132475 & 6.12 & -1.38 & 5.97 & -1.23 & 0.15 \\
\hline HD 201891 & 6.47 & -1.03 & 6.26 & -0.94 & 0.09 \\
\hline HD 201889 & 6.79 & -0.71 & 6.69 & -0.51 & 0.20 \\
\hline
\end{tabular}

\subsection{Sulfur and iron abundances}

The resulting sulfur abundances for our stars are presented in Table 5 as $\log A_{\mathrm{S}}$, the $\log$ arithmic abundance relative to the solar value of $\log A_{\mathrm{S}, \odot}=7.20$, i.e. $[\mathrm{S} / \mathrm{H}]$, and the logarithmic abundance relative to the solar value normalized to the metallicity ([S/Fe]). Also, displayed in the Table are the iron abundances calculated from the measured equivalent widths of Fe II lines shown in Table 2. The total uncertainty, including effects of statistical measuring-uncertainties and uncertainties in the model parameters, is estimated to be of the order of \pm 0.15 dex.

In their non-LTE analysis of neutral sulfur in environments relevant also in our study, TH find inconsequential effects for the $\lambda \lambda 8694-95$ lines, ranging from 0.00 to $0.08 \mathrm{dex}$ in the sulfur abundance. Nissen et al. (2003b) find in their study of the sulfur abundance for similar stars, that the sulfur abundance they derive from the $\lambda \lambda 8694-95$ lines match the abundance derived from the $\lambda \lambda 9213-38$ lines very well, indicating that departures from LTE should be small also for the latter lines. It should be noted that neutral sulfur atoms represent the main ionization state throughout most of the atmosphere, and most importantly in the line-forming regions. Thus, we judge that the NLTE effects should be small, justifying a standard LTE analysis. Furthermore, Nissen et al. (2003a) investigate and find that the $3 \mathrm{D}$ effects ${ }^{2}$ for dwarfs will not severely alter the $[\mathrm{S} / \mathrm{Fe}]$ ratio (less than $0.05 \mathrm{dex}$ ). Therefore, we will neither make any corrections for non-LTE effects nor for 3D effects. Our [S/Fe] values are plotted versus $[\mathrm{Fe} \mathrm{II} / \mathrm{H}]$ in Fig. 4. It is clear that departures from LTE and the 3D effects have very similar (small) effects on the abundance derived from the $\lambda \lambda 9213-38$ lines and that from the $\lambda \lambda 8694-95$ lines.

Since sulfur abundances have been obtained previously for each of our stars using the $\lambda \lambda 8694-95$ lines, it is of considerable interest to compare $\mathrm{S}$ (and Fe) abundances. This comparison is made in Tables 6 and 7.

\footnotetext{
${ }^{2}$ By "3D effects" is meant differences in, for example, derived abundances when using atmospheric models including 3D, hydrodynamic modelling of the convection compared with traditional atmospheric models, which describe convection by the mixing-length approximation.
}

Table 6. Sulfur abundances compared to those from the literature which are based on the $\lambda \lambda 8694-95$ lines.

\begin{tabular}{|c|c|c|c|}
\hline Star & $\begin{array}{c}\log A_{\mathrm{S}} \\
\lambda \lambda 9213-38 \text { lines } \\
\text { this work }\end{array}$ & $\begin{array}{c}\log A_{\mathrm{S}} \\
\lambda \lambda 8694-95 \text { lines } \\
\text { literature }\end{array}$ & reference \\
\hline \multicolumn{4}{|c|}{ Giants and subgiants } \\
\hline $\begin{array}{l}\text { HD } 2665 \\
\text { HD } 88609 \\
\text { HD } 111721 \\
\text { HD } 165195\end{array}$ & $\begin{array}{l}5.60 \\
4.74 \\
6.25 \\
5.97\end{array}$ & $\begin{array}{c}5.89 \\
<5.49 \\
6.3 / \mathbf{6 . 0 2}^{a} /-^{b} \\
5.98\end{array}$ & $\begin{array}{l}(1) \\
(2) \\
(3) \\
(2)\end{array}$ \\
\hline \multicolumn{4}{|l|}{ Dwarfs } \\
\hline $\begin{array}{l}\text { HD } 19445 \\
\text { HD } 84937 \\
\text { HD } 94028 \\
\text { HD } 132475 \\
\text { HD } 201891 \\
\text { HD } 201889\end{array}$ & $\begin{array}{l}5.50 \\
5.27 \\
5.93 \\
5.97 \\
6.26 \\
6.69\end{array}$ & $\begin{array}{c}5.97 \\
5.70 \\
6.3 / \mathbf{6 . 0 6}^{a} / 6.09^{b} \\
6.4 / \mathbf{6 . 0 0}^{a} / 6.08^{b} \\
6.6 / \mathbf{6 . 2 9}^{a} / 6.33^{b} \\
6.83\end{array}$ & $\begin{array}{l}(1) \\
(2) \\
(3) \\
(3) \\
(3) \\
(1)\end{array}$ \\
\hline
\end{tabular}
(1) Israelian \& Rebolo (2001).
(2) Takada-Hidai et al. (2002).
(3) François (1988).

a A reanalysis by Takada-Hidai et al. (2002) using François's (1988) equivalent widths.

${ }^{b}$ The corresponding reanalysis by Israelian \& Rebolo (2001).

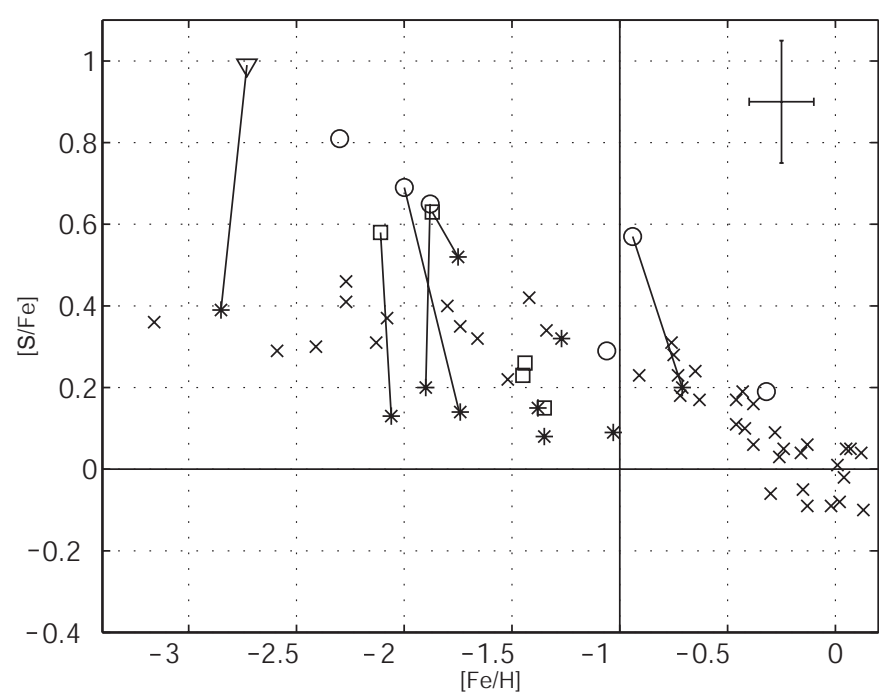

Fig. 4. This figure shows the Galactic chemical evolution of sulfur. The iron and sulfur abundances, as we have determined it from analyzing Fe II lines and sulfur lines in the NIR, are presented with star symbols. Typical errorbars for our measurements of $\pm 0.15 \mathrm{dex}$, both for $[\mathrm{S} / \mathrm{Fe}]$ and $[\mathrm{Fe} / \mathrm{H}]$, are indicated. Circles indicate the sulfur and iron abundances derived by Israelian \& Rebolo (2001) and squares display values found by Takada-Hidai et al. (2002) (including an upper limit which is illustrated with a triangle). For the 6 star we have in common with Israelian \& Rebolo (2001) and Takada-Hidai et al. (2002), we have connected our new determinations with their measurements, which are based on the sulfur $\lambda \lambda 8694-95$ lines. We have also plotted sulfur abundances derived by Nissen et al. (2003a) and Chen et al. (2002) for southern stars. These are plotted with crosses. 
Table 7. The iron and sulfur abundance ratios $([\mathrm{Fe} / \mathrm{H}],[\mathrm{S} / \mathrm{H}]$, and $[\mathrm{S} / \mathrm{Fe}])$ from our work and from the literature for the $\lambda \lambda 8694-95$ lines.

\begin{tabular}{|c|c|c|c|c|c|c|c|}
\hline \multirow[t]{2}{*}{ Star } & \multicolumn{2}{|c|}{$[\mathrm{Fe} / \mathrm{H}][\mathrm{S} / \mathrm{H}]$} & {$[\mathrm{S} / \mathrm{Fe}]$} & {$[\mathrm{Fe} / \mathrm{H}]$} & {$[\mathrm{S} / \mathrm{H}]$} & {$[\mathrm{S} / \mathrm{Fe}]$} & \multirow[t]{2}{*}{ reference } \\
\hline & \multicolumn{3}{|c|}{ based on the $\lambda \lambda 9213-38$ lines } & \multicolumn{3}{|c|}{ study based on the $\lambda \lambda 8694-95$ lines } & \\
\hline \multicolumn{8}{|c|}{ Giants and subgiants } \\
\hline HD 2665 & -1.74 & -1.60 & 0.14 & -2.0 & $-1.31 \pm 0.14$ & 0.69 & (1) \\
\hline HD 88609 & -2.85 & -2.46 & 0.39 & -2.73 & $<-1.72$ & $<0.99$ & (2) \\
\hline HD 111721 & -1.27 & -0.95 & 0.32 & $-1.57 /-\mathbf{1 . 2 9}^{a} /-$ & $-0.86 /-\mathbf{1 . 1 9}^{\mathrm{a}} /-$ & $0.71 / \mathbf{0 . 0 7} \pm \mathbf{0 . 4 1}^{a} /-$ & (3) \\
\hline HD 165195 & -1.75 & -1.23 & 0.52 & -1.87 & -1.23 & $0.64 \pm 0.09$ & (2) \\
\hline \multicolumn{8}{|l|}{ Dwarfs } \\
\hline HD 19445 & -1.90 & -1.70 & 0.20 & -1.88 & $-1.23 \pm 0.12$ & 0.65 & (1) \\
\hline HD 84937 & -2.06 & -1.93 & 0.13 & -2.11 & -1.51 & $0.60 \pm 0.06$ & (2) \\
\hline HD 94028 & -1.35 & -1.27 & 0.08 & $-1.48 /-\mathbf{1 . 2 6}^{a} /-1.31^{b}$ & $-0.91 /-\mathbf{1 . 1 5}^{a} /-1.12^{b}$ & $0.57 / \mathbf{0 . 1 1}^{a} / 0.19^{b}$ & (3) \\
\hline HD 132475 & -1.38 & -1.23 & 0.15 & $-1.37 /-\mathbf{1 . 2 3}^{a} /-1.37^{b}$ & $-0.81 /-\mathbf{1 . 2 1}^{a} /-1.13^{b}$ & $0.56 / \mathbf{0 . 0 1}^{a} / 0.24^{b}$ & (3) \\
\hline HD 201891 & -1.03 & -0.94 & 0.09 & $-1.25 /-\mathbf{0 . 9 8}^{a} /-0.87^{b}$ & $-0.61 /-\mathbf{0 . 9 2} 2^{a} /-0.88^{b}$ & $0.64 / \mathbf{0 . 0 5}^{a} /-0.01^{b}$ & (3) \\
\hline HD 201889 & -0.71 & -0.51 & 0.20 & -0.94 & $-0.37 \pm 0.1$ & 0.57 & (1) \\
\hline
\end{tabular}
(1) Israelian \& Rebolo (2001).
(2) Takada-Hidai et al. (2002).
(3) François (1988).

a A reanalysis by Takada-Hidai et al. (2002) using François's (1988) equivalent widths.

$b$ The corresponding reanalysis by Israelian \& Rebolo (2001).

Three stars - one giant and two dwarfs - are in common with I\&R. Our sulfur abundances are systematically smaller: $\Delta \log A_{\mathrm{S}}=\log A_{\mathrm{S}}$ (ours) $-\log A_{\mathrm{S}}$ (theirs) $=-0.29$ (HD 2665), -0.47 (HD 19445), and -0.14 (HD 201889). Recall that we have used the same stellar parameters. It is likely to be significant that the agreement is best for the star (HD 201889) for which the $\lambda \lambda 8694-95$ lines are strongest. The difference is in fact -0.14 dex also when the abundance of I\&R is corrected to the NIST $g f$-value (abundance increased by $0.02 \mathrm{dex}$ ) and to the MARCS model (abundance decreased by $0.02 \mathrm{dex}$ ). We suppose that the larger differences for the two more metal-poor stars reflect the greater uncertainty in measuring very weak S I 28695 line (equivalent widths of about $3 \mathrm{~m} \AA$ ). Alternative choices of model atmosphere parameters cannot erase the abundance difference resulting from the choice of S I lines. The corresponding $\Delta s$ for $[\mathrm{Fe} / \mathrm{H}]$ are +0.26 (HD 2665), -0.02 (HD 19445), and +0.23 (HD 201889). I\&R indicate that the iron abundance determination is based on $\mathrm{Fe} I$ lines and a nonLTE analysis. We greatly prefer to derive the $\mathrm{Fe}$ abundance from Fe II lines, for which non-LTE effects are slight, than to use $\mathrm{Fe} \mathrm{I}$ lines and an uncertain non-LTE correction. Fe II lines are, however, more sensitive than $\mathrm{Fe} I$ lines to uncertainties in the surface gravity, but this is also true for $\mathrm{S}$ I lines compared to $\mathrm{S}$ II. Thus, this sensitivity should cancel out in the $\mathrm{S} \mathrm{I} / \mathrm{Fe}$ II ratio.

There are large differences in the $[\mathrm{S} / \mathrm{Fe}]$ ratios obtained by us and I\&R: 0.14 vs. 0.69 (HD 2665), 0.20 vs. 0.65 (HD 19445), and 0.20 vs. 0.57 (HD 201889) arising from a higher $\mathrm{S}$ abundance in each case and a lower $\mathrm{Fe}$ abundance in two cases. In short, we do not confirm the high $[\mathrm{S} / \mathrm{Fe}]$ proposed by $I \& R$.
Three of our stars were observed and analyzed previously by TH. For HD 88609 , the most metal-poor star $([\mathrm{Fe} / \mathrm{H}]=$ -2.8 ) of the trio, their upper limit to the $\mathrm{S}$ abundance is a safe 0.7 dex higher than our measured abundance. For HD 84937 with $[\mathrm{Fe} / \mathrm{H}]=-2.1$, the sulfur abundance derived by $\mathrm{TH}$ is 0.45 dex larger than ours but this difference is probably due to the uncertain equivalent width of the $\lambda 8695$ line; TH note that their spectrum is of low signal-to-noise ratio and marred by residual fringing. In the case of HD 165195, our and their S abundances agree well. These comparisons use LTE abundances derived by TH; the corrections for non-LTE effects are small. The LTE Fe abundances derived by TH from a set of Fe II lines are in good agreement with ours: $\Delta \mathrm{s}$ are $-0.12,+0.12$, and +0.05 . The $[\mathrm{S} / \mathrm{Fe}]$ values for the two stars for which $\mathrm{TH}$ detected the $\lambda 8695$ line in the sense ours vs. theirs, are 0.52 vs. 0.64 (HD 165195) and 0.13 vs. 0.60 (HD 84937). TH detected the $\lambda \lambda 8694-95$ lines in three stars not observed by us. Their detections appear quite secure. We note that the $[\mathrm{S} / \mathrm{Fe}]$ ratio inferred by $\mathrm{TH}$ for these stars, with $[\mathrm{Fe} / \mathrm{H}] \simeq-1.4$, average about 0.2 and fit well with our suggested run of $[\mathrm{S} / \mathrm{Fe}]$ with $[\mathrm{Fe} / \mathrm{H}]$, see Fig. 4.

We chose four stars from François (1988). This quartet was reanalysed by $\mathrm{TH}$ and I\&R using equivalent widths of the $\lambda 8695$ line reported by François (1988). Table 6 shows that, for the dwarfs, these reanalyses and our abundance from the NIR lines are in good agreement. The agreement extends to $[\mathrm{S} / \mathrm{Fe}]$, cf. Table 7 . The typical total uncertainties quoted by $\mathrm{TH}$, for their dwarfs, for the $[\mathrm{S} / \mathrm{Fe}]$ ratio is $0.15 \mathrm{dex}$. However, for their analysis of HD 111721 the uncertainty is larger ( 0.4 dex). 


\section{Discussion}

Our observations show that the S I NIR lines are detectable and $\mathrm{S}$ abundances determinable to $[\mathrm{Fe} / \mathrm{H}] \simeq-3$, and, thus, confirm the conclusions of Nissen et al. (2003a) from their VLT/UVES observations of the same lines in southern dwarfs and subgiants. In Fig. 4, we show our results with those of Nissen et al. (2003a) for halo stars, also based on the $\lambda \lambda 9213-38$ lines, and Chen et al. (2002) for disk stars. Chen et al. (2002) used the $\lambda \lambda 8694-95$ lines and also lines at 6046, 6053, and $6757 \AA$, but for their chosen disk stars these lines provide readily measurable lines in high-resolution spectra. In Fig. 4, our values for the stars in common with I\&R and TH are connected with theirs to guide the eye. For the stars we have in common with $\mathrm{TH}$, we have, for consistency, chosen their $[\mathrm{FeII} / \mathrm{H}]_{\mathrm{LTE}}$ and $[\mathrm{S} / \mathrm{FeII}]_{\mathrm{LTE}}$ values. The figure illustrates the comparisons between our and the $\lambda \lambda 8694-95$-based abundances discussed in the previous section.

There is no evidence in our data for the rise of $[\mathrm{S} / \mathrm{Fe}]$ with decreasing $[\mathrm{Fe} / \mathrm{H}]$ earlier proposed by I\&R and TH. I\&R claimed a linear trend such that $[\mathrm{S} / \mathrm{Fe}] \simeq 0.9$ at $[\mathrm{Fe} / \mathrm{H}]=-2$, and $\mathrm{TH}$ put the slope lower so that $[\mathrm{S} / \mathrm{Fe}]=0.5$ at $[\mathrm{Fe} / \mathrm{H}]=$ -2 . We have suggested that the lower $[\mathrm{S} / \mathrm{Fe}]$ now being obtained are due to a combination of overestimates of the strength of the very weak $\lambda \lambda 8694-95$ lines in the most metal-poor stars, and of underestimates of the Fe abundances by I\&R. We reiterate that the adopted effective temperatures and surface gravities are those used by I\&R and TH. Our primary goal was to check the latter authors' $\mathrm{S}$ abundances, as directly as possible, by using $\lambda \lambda 9213-38$ lines rather than the $\lambda \lambda 8694-95$ lines used by them. Definition of the run of $[\mathrm{S} / \mathrm{Fe}]$ with $[\mathrm{Fe} / \mathrm{H}]$ was a secondary goal whose full achievement demands additional stars. As can be seen in Fig. 4, the evidence that $[\mathrm{S} / \mathrm{Fe}]$ is nearly independent of $[\mathrm{Fe} / \mathrm{H}]$ for $[\mathrm{Fe} / \mathrm{H}] \lesssim-1$ is greatly strengthened on adding the sulfur abundances derived by Nissen et al. (2003a), who find a constant value of $[\mathrm{S} / \mathrm{Fe}] \approx 0.35 \mathrm{dex}$.

There is a hint in Fig. 4 that our $[\mathrm{S} / \mathrm{Fe}]$ estimates may be systematically about 0.1 dex lower than those of Nissen et al. (2003a). This difference could be the result of an accumulation of minor differences in effective temperature scales, surface gravity determinations, Fe II $g f$-values, and others. We also note, that Nissen et al. (2003a) derive a solar iron abundance 0.03 dex larger than the one we apply. Considering this fact would diminish the discrepancy.

Many studies of abundance of $\alpha$ elements in halo stars have shown that $[\alpha / \mathrm{Fe}]$ at a fixed $[\mathrm{Fe} / \mathrm{H}]$ is largely without cosmic scatter. The $\mathrm{S}$ abundances of Nissen et al. (2003a) confirm that this result may now be extended to sulfur. In our small sample, HD 165195 appears to depart from the constant $[\mathrm{S} / \mathrm{Fe}]$ indicated by other stars. This departure may possibly be due to adoption of incorrect atmospheric parameters. We note that $\mathrm{TH}$ remark that the determination of the effective temperature for HD 165195 is uncertain. They consider a range of temperatures (4131-4507 K) in which their adopted temperature (and therefore the one used by us) is at the lower end of this range. Observe, that the sulfur abundances determined for this star are the most sensitive to the effective temperature of our entire sample, which can be seen in Table 4. A temperature increase of $300 \mathrm{~K}$ would yield a [S/Fe] ratio close to our mean value. Therefore, our value might be seen as a determination which could be on the high side. We note, in passing, that an increase in temperature would also make the modelled hydrogen-line fit the observed line better.

The exact run of the abundances with metallicity has consequences for theories and our understanding of Galactic chemical evolution and for the sites of formation of the elements. So, what are the sites where sulfur was synthesized during the first few billion years of the Universe? $\alpha$-capture elements are thought to be formed during explosions of supernovae $(\mathrm{SNe})$ type II. Owing to the short life-times of high-mass stars, the progenitors of SNe type II, the abundances of elements synthesized by them and ejected into the interstellar medium, quickly reach a steady state and show a constant over-abundance in the ordinary $[\mathrm{S} / \mathrm{Fe}]$ vs. $[\mathrm{Fe} / \mathrm{H}]$ diagram. However, based on their data, TH and I\&R discern a linear rise of the $\alpha$-capture element sulfur for the halo phase of the Milky Way, thus showing a divergent behaviour compared to other $\alpha$-capture elements, except possibly oxygen. If there is a rise in the abundances with decreasing metallicity, other processes for their formation have to be invoked. TH and I\&R suggest a sulfur contribution from hypernovae in the early galaxy as an explanation of the high values. Hypernovae are thought to be the explosion of an extremely massive star (several $100 M_{\odot}$ ). Another mechanism that could lead to a divergent evolutionary behaviour of oxygen and sulfur as compared to the other $\alpha$-capture elements, is the fact that these two elements are volatile elements as opposed to the others which are refractory elements. This is important in the modelling of the transport and mixing of supernovae ejecta into the interstellar medium, with volatile elements experiencing a mixing time-scale, more than an order of magnitude faster. However, considering the results that Nissen et al. (2003a) and we find, there does not seem to be a need for a distinct evolutionary scenario for sulfur as compared to the other $\alpha$ elements.

\section{Conclusions}

We have shown that reliable abundances of sulfur down to metallicities of $[\mathrm{Fe} / \mathrm{H}] \sim-3$ are obtainable using the $\mathrm{S}$ I lines lying at 9212.9, 9228.1, and 9237.5 А. In an attempt to resolve the discrepancy found in the literature, about the Galactic chemical evolution of sulfur for metallicities lower than $[\mathrm{Fe} / \mathrm{H}] \lessgtr-1$, we have observed an ensemble of ten stars, both dwarfs and giants, previously analyzed with a different and weaker diagnostic; the S I lines at 8694.0 and $8694.6 \AA$. These previous investigations of the 10 stars claimed a rise of the $[\mathrm{S} / \mathrm{Fe}]$ ratio with decreasing metallicity. We are not able to confirm this rise. Our conclusion is instead that we corroborate the finding by Nissen et al. (2003a) indicating a similar behaviour of sulfur to the other $\alpha$ elements.

We suggest that the reasons for the difference in determined sulfur abundances and $[\mathrm{S} / \mathrm{Fe}]$ are the strength of the lines analyzed and the way the metallicity is determined. First, we have used lines which are stronger by a factor of ten as compared to those used earlier. This is critical for metal-poor stars. Thus, we conclude that the smallest equivalent widths for the 
weaker $\lambda 8695$ line must have been overestimated in the previous studies, leading to an overestimation of the $[\mathrm{S} / \mathrm{H}]$ ratio by up to 0.5 dex. Second, we have determined the metallicity from Fe II lines which should give a better determination of the metallicities than an non-LTE analysis of Fe I lines.

We also show that in spite of the numerous telluric, watervapor lines in the $9200 \AA$ spectral region, a careful reduction of the data can provide clean spectra. We conclude that the $9212.9,9228.1$, and $9237.5 \AA$ lines are the preferred ones to be used for abundance analyses of sulfur of halo stars.

Acknowledgements. We should like to thank Drs. C. Allende Prieto, B. Gustafsson, K. Eriksson, B. Edvardsson, A. Kron, and N. Piskunov for valuable discussions and comments. This research has been supported in part by the Swedish Research Council, Stiftelsen Blanceflor Boncompagni-Ludovisi, née Bildt, the Swedish Foundation for International Cooperation in Research and Higher Education, and the Robert A. Welch Foundation of Houston, Texas.

\section{References}

Alonso, A., Arribas, S., \& Martinez-Roger, C. 1996, A\&A, 313, 873 Alonso, A., Arribas, S., \& Martínez-Roger, C. 1999a, A\&AS, 140, 261
Alonso, A., Arribas, S., \& Martínez-Roger, C. 1999b, A\&AS, 139, 335

Chen, Y. Q., Nissen, P. E., Zhao, G., \& Asplund, M. 2002, A\&A 390, 225

Edvardsson, B., Andersen, J., Gustafsson, B., et al. 1993, A\&A, 275, 101

François, P. 1988, A\&A, 195, 226

Gray, D. F. 1992, The observation and analysis of stellar photospheres (Cambridge; New York: Cambridge University Press), 2nd ed.

Gustafsson, B., Bell, R. A., Eriksson, K., \& Nordlund, Å. 1975, A\&A, 42, 407

Israelian, G., García López, R. J., \& Rebolo, R. 1998, ApJ, 507, 805

Israelian, G., \& Rebolo, R. 2001, ApJ, 557, L43

Nissen, P. E., Chen, Y. Q., Asplund, M., \& Pettini, M. 2003a, in Modelling of Stellar Atmospheres, ed. N. Piskunov, \& W. Weiss, IAU Symp., 210, in press

Nissen, P. E., Chen, Y. Q., Asplund, M., \& Pettini, M. 2003b, A\&A, submitted

Piskunov, N. E., Kupka, F., Ryabchikova, T. A., Weiss, W. W., \& Jeffery, C. S. 1995, A\&AS, 112, 525

Ryan, S. G., \& Lambert, D. L. 1995, AJ, 109, 2068

Takada-Hidai, M., Takeda, Y., Sato, S., et al. 2002, ApJ, 573, 614

Thévenin, F., \& Idiart, T. P. 1999, ApJ, 521, 753

Thorén, P., \& Feltzing, S. 2000, A\&A, 363, 692

Tull, R. G., MacQueen, P. J., Sneden, C., \& Lambert, D. L. 1995, PASP, 107, 251 